\title{
Platooning of Car-like Vehicles in Urban Environments: Longitudinal Control Considering Actuator Dynamics, Time Delays, and Limited Communication Capabilities
}

\author{
Ahmed Khalifa, Olivier Kermorgant, Salvador Dominguez, and Philippe Martinet
}

\begin{abstract}
This paper proposes a longitudinal control framework for platooning in an urban environment. The targetted application is to redistribute vehicles involved in car-sharing systems, where only the leader vehicle is human-driven. We propose a platoon model and control law considering actuator dynamics. This control relies on a hybrid Information Flow Topology (IFT), where the leader broadcasts its state but each follower only measures the position of its predecessor. A consensusbased control law incorporates the effect of the network/sensor time delay and the variable velocity of the leader. Conditions for the platoon internal and string stability are given. Experiments demonstrate the efficiency of the framework in simulation and real experiments with three commercial cars.
\end{abstract}

Index Terms-Longitudinal control, platoon, curvilinear coordinates, hybrid information flow topology, string stability

\section{INTRODUCTION}

Shared transportation systems in urban environments are the current trend toward an eco-friendly city. In car-sharing systems, one issue is to ensure the cars are always well distributed in all stations. A potential solution that we investigate is to have a single human-driven vehicle able to pick and place autonomous cars. The driver can easily communicate highlevel orders (typically unpark, join, merge, follow, split, park) to the autonomous cars, that will then activate the suitable behavior. In this work, we assume the platoon is already set up and we focus on the distributed platoon control.

Platooning consists in following a leader vehicle's path, with constraints on the inter-vehicle distances. This distance is usually assumed to be constant as it achieves a high traffic capacity [1]. The platoon should be string stable, meaning that the error signals attenuate when propagating downstream the platoon. String instability may result in a rear-end collision. An efficient way to model the cars and the control law is the path coordinates as it allows decoupling the longitudinal and lateral control laws as illustrated in Fig. 1. Besides, distances between vehicles are monotone along the path, while they are nonmonotone if using a Cartesian description [2]. In the sequel,

The authors are with the Laboratoire des Sciences du Numérique de Nantes (LS2N), Ecole Centrale de Nantes, 1 rue de la Noë, 44321 Nantes, France, given-name.namedec-nantes. fr.

A. Khalifa is on leave from the Dep. of Industrial Electronics and Control Eng., Faculty of Electronic Engineering, Menoufia University, Egypt.

P. Martinet is with Inria Sophia Antipolis, 06902 Sophia Antipolis, France, philippe.martinet@inria.fr. we assume a suitable control [3] is used for the lateral control, that steers the vehicle to stay on the leader's path.

The goal of the longitudinal control is to output the required throttle or brake that ensures the desired behavior is performed along the path. Existing works focus on Vehicle Dynamics, Distributed Controller and Information Flow Topology [4].

Nonlinear vehicle dynamic models [5], [6] make it difficult to analyze the system performance against different spacing policies and communications typologies. Linear models are thus frequently used. The simplest ones are single integrator [7] or double integrators [8], [9]. These approaches ignore some of the vehicle dynamics which may affect the system performance in real-time experiments. In third order models, the power-train model is also considered [10]. An originality of our approach is to consider a third order model for longitudinal dynamics, expressed in the path coordinates. To the best of our knowledge, path coordinates have mostly be used with a first-order model for the vehicle dynamics [2], [11], [12]. Distributed control [13] assumes some information sharing between vehicles to improve performance. These controllers can be linear [14], optimal [15], sliding Mode [16], modelpredictive [17], and consensus-based controller [9], [10], [18], [19]. The main drawback of existing works is that most of them assume that the leader travels with a constant velocity, which is usually valid for highways but not for urban environments. In this study, we consider a potentially highly varying leader velocity. Besides, for the sake of simplicity the longitudinal control is expressed in path coordinates.

The Predecessor-Leader Following (PLF) topology [1] corresponds to all vehicles receiving the state of the leader and of their direct predecessor. An originality of our approach is to assume all vehicles carry a LiDAR for localization in an urban environment. We thus propose a hybrid PLF topology where the leader broadcasts its state, but a follower only retrieves the position of its predecessor through LiDAR. This is wellsuited for urban scenarios where the path can be highly curved and where a radar would have too narrow a field of view. The proposed control law thus only relies on the measured predecessor's position. A low-cost communication module can hence be used to receive information from the leader.

The main contributions of the proposed solution with respect to previous works are thus:

- A control algorithm is designed considering varying velocity and high curvature and hybrid PLF topology 


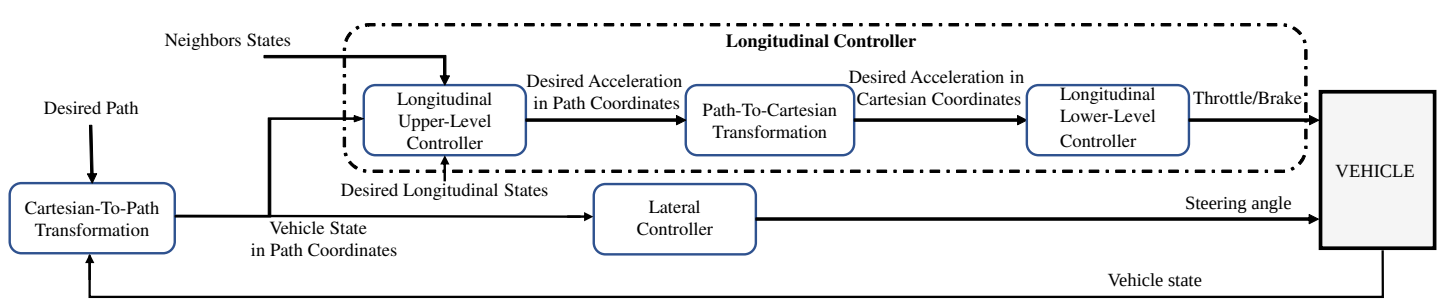

Figure 1. Functional block diagram of the vehicle control sub-layer.

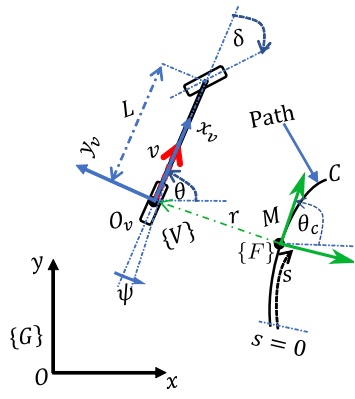

Figure 2. Kinematic model of vehicle. $r$ is negative in this example.

- Conditions for both internal and string stability are provided under the effect of communication and sensor delay and actuator dynamics.

- The proposed framework is validated via a realistic simulation and experiments with commercial cars.

This work builds on the preliminary results presented in [20]. The major improvements is to consider actuator dynamics, and to provide realistic simulations and real experiments.

The paper is organized as follows: Section II formulates introduces the notations for the kinematic and dynamic models. In Section III the proposed control law is presented, and both internal and string stability are analyzed. Simulation and experimental results are given in Sections IV.

\section{Problem Statement}

\section{A. Vehicle Kinematic Model}

The kinematic model of a vehicle can be simplified to that of a bicycle model [3], see Fig. 2, in which the left and right wheels are combined into a pair of single wheels at the center of the front and rear axles.

1) Cartesian Coordinates Model: The configuration of the vehicle, with respect to the world-fixed frame, $\{G\}, O-x y$, is represented by the generalized coordinates $[x, y, \theta]^{T} \in \mathbb{R}^{3}$, where its position (center $O_{v}$ of the rear axle) is given by $[x, y]^{T}$ and its orientation in the global frame is represented by $\theta . \delta$ is the steering angle. The kinematic model is given by

$$
\begin{aligned}
\dot{x} & =v \cos (\theta), \\
\dot{y} & =v \sin (\theta), \\
\dot{\theta} & =\frac{v}{L} \tan (\delta) .
\end{aligned}
$$

2) Path Coordinates Model: Let us define the curvilinear coordinates $[s, r, \psi]^{T}$, as shown in Fig. 2. The tracking path $C$, defined in the Global Frame, can be represented as a function of its length $s$ (curvilinear abscissa) at the closest point $M$ to $O_{v}$, the relative angle (angular deviation), $\psi=\theta-\theta_{c}$, of the vehicle with respect to the path, where $\theta_{c}$ is the angle between the path tangent at $M$ and the $x$-axis, and finally, the lateral deviation, $r$, which is the signed distance, expressed in the Frenet frame $\{F\}$ (green in Fig. 2) from the center of the rear axle $O_{v}$, to the closest point on the path $M$. The kinematic model in the path coordinates is thus given by

$$
\begin{aligned}
\dot{s} & =v \frac{\cos (\psi)}{1-r \kappa(s)}, \\
\dot{r} & =v \sin (\psi), \\
\dot{\psi} & =v\left(\frac{\tan (\delta)}{L}-\frac{\kappa(s) \cos (\psi)}{1-r \kappa(s)}\right),
\end{aligned}
$$

where $\kappa(s)$ is the curvature of path at point $M$.

\section{B. Vehicle Longitudinal Dynamic Model}

The dynamic model is presented assuming:

Assumption 1. The vehicle body is rigid and symmetric.

Assumption 2. The driving and braking inputs are integrated into one control input.

Assumption 3. Pitch and yaw motions are neglected.

Assumption 4. The power-train dynamics are lumped to be a first-order inertial transfer function.

Therefore, longitudinal dynamics can be represented by

$$
\begin{aligned}
M_{v} a+C_{v} v+G_{v} & =F_{v}, \\
\tau \dot{F}_{v}+F_{v} & =F_{v, \text { des }}
\end{aligned}
$$

where $\tau$ is a parameter characterizing the actuator dynamics, $a=\dot{v}$ denotes the vehicle acceleration in the vehicle frame, and $M_{v}, C_{v}, G_{v}$, and $F_{v}$ are the vehicle Inertia effect, Coriolis effect $^{1}$, Gravity effect, and input force, respectively.

The inverse model compensation technique is frequently used to eliminate the non-linearities in longitudinal dynamics for the purpose of high-level control design. The control law of the inverse dynamics technique is given by

$$
F_{v, d e s}=M_{v} \mu+C_{v}(\tau a+v)+G_{v}
$$

where $\mu$ is the new input signal after system linearization which has to be designed. By replacing $F_{v}$ and $\dot{F}_{v}$ from (3a)

\footnotetext{
${ }^{1}$ Note that for autonomous cars, the Coriolis effect can be neglected as Earth can be considered flat with regards to the involved velocities.
} 


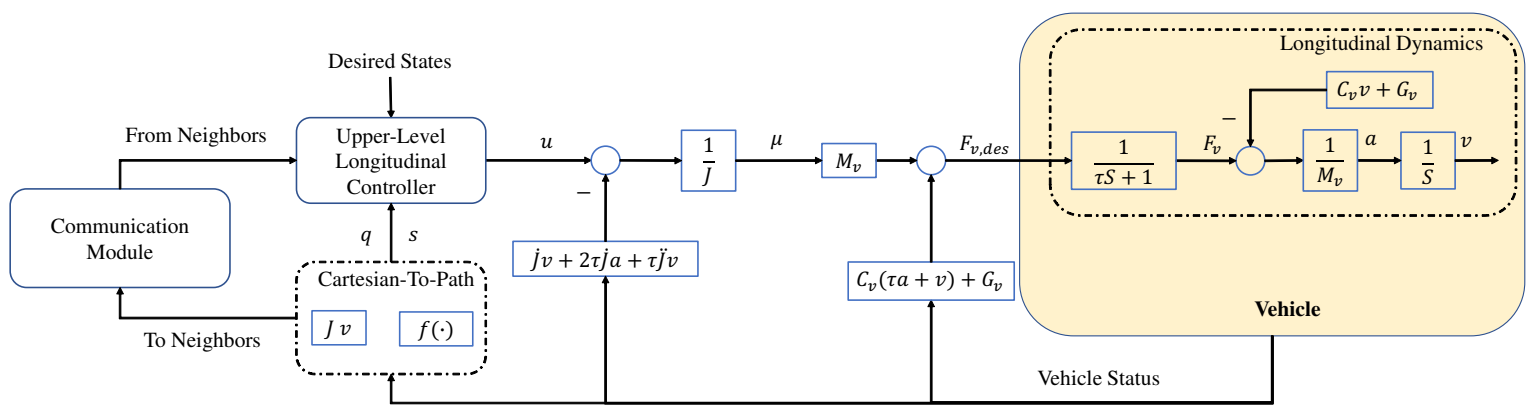

Figure 3. Block diagram of the vehicle longitudinal dynamic model, where $S$ is the Laplace operator.

and its time-derivative, and $F_{v, \text { des }}$ from (4) into (3b), we get the third order dynamic model in the body coordinates:

$$
\tau \dot{a}+a=\mu
$$

We now detail the third-order model expressed in the path coordinates. The corresponding controller is given in Fig. 3.

Let us recast $(2 a)$ as

$$
\dot{s}=J v
$$

where $J$ is given by

$$
J=\frac{\cos (\psi)}{(1-r \kappa)} .
$$

Thus, the acceleration in the path coordinates, $\eta$, is given by

$$
\eta=J a+\dot{J} v
$$

where $\eta=\dot{q}=\ddot{s}$ and $q=\dot{s}$ are the vehicle acceleration and velocity in the path coordinates respectively. If the mapping from the control signal (i.e., desired acceleration) in the path coordinates, $u$, to that in the body coordinates, $\mu$, is given by differentiating (8) with regards to time:

$$
\mu=\frac{1}{J}(u-\dot{J} v-2 \tau \dot{J} a-\tau \ddot{J} v),
$$

then, the longitudinal dynamics in the path coordinates are

$$
\begin{aligned}
\dot{s} & =q, \\
\dot{q} & =\eta, \\
\tau \dot{\eta}+\eta & =u .
\end{aligned}
$$

In practice, $\dot{J}$ is obtained by differentiating (7) with regards to time, and $\ddot{J}$ is computed by numerical differentiation. As $\tau \ddot{J} v$ is a small term, the approximation does not affect the behavior of the system.

\section{Platoon Longitudinal Model}

Consider a platoon with $N+1$ vehicles, where the leader has index 0 and $N$ followers have indices $i ; i \in[1, N]$ (see Fig. 4). The vehicles share information by either sensor-based or communication-based link. The vehicle dynamics in the path coordinates are given by (10) and we now denote $(.)_{i}$ the variables related to vehicle $i$.

The inter-vehicle distance, $d_{i}=s_{i-1}-s_{i}$, is the curvilinear distance between vehicle $i$ and its predecessor, $d_{i 0}=s_{0}-s_{i}$, is the curvilinear distance between vehicle $i$ and leader, $d_{r, i}$ is

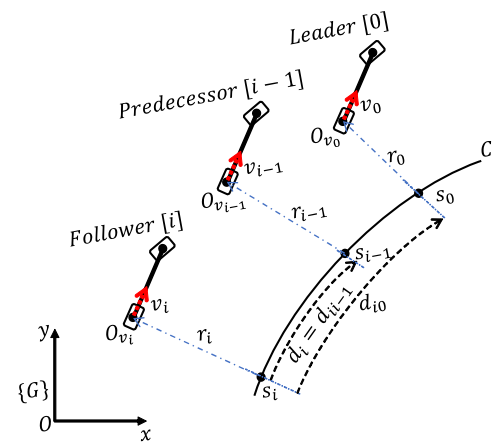

Figure 4. Platoon representation in the path coordinates.

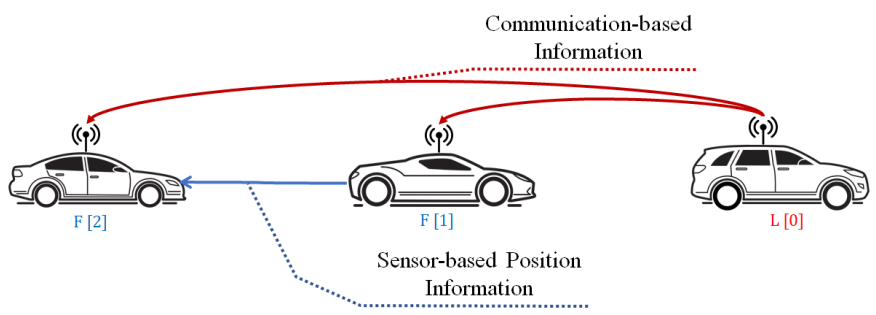

Figure 5. Information flow topology: Hybrid PLF through communication link between leader and followers and direct sensor-based measurements between followers.

the desired inter-vehicle distance between vehicles $i$ and $i-1$, and $d_{r, i 0}$ is the desired inter-vehicle distance between vehicles $i$ and 0 .

\section{Control Objectives}

The platoon has to track a leader that is either autonomous or manually driven, and each follower vehicle, $i$, has the following delayed information, see Fig. 5:

- position, velocity, and acceleration of the leader, $s_{0}, q_{0}, \eta_{0}$;

- position of its predecessor $s_{i-1}$ due to the considered limited communication capability.

Under these conditions, $\forall i=1, \ldots, N$, the following goals have to be achieved:

- asymptotic stability of the position and velocity tracking;

- string stability.

\section{Control Design}

This section introduces the proposed control algorithms. Internal stability and string stability are then analyzed. 


\section{A. Consensus-based Control Law}

The information flow of the platoon is described by an adjacency matrix $\mathcal{M}=\left[m_{i j}\right]$, where $m_{i j}=1$ if vehicle $i$ can obtain information from vehicle $j$, otherwise $m_{i j}=0$. For the Predecessor - Leader Following topology, $\mathcal{M}$ is defined as

$$
m_{i j}= \begin{cases}1, & j=0 \text { or } j=i-1 \\ 0, & \text { otherwise. }\end{cases}
$$

The Laplacian matrix $\mathcal{L} \in \mathbb{R}^{N \times N}$ associated with $\mathcal{M}$ is

$$
\mathcal{L}=\left[l_{i j}\right] \text { where } l_{i j}= \begin{cases}-m_{i j}, & i \neq j, \\ \sum_{k=1}^{N} m_{i k}, & i=j .\end{cases}
$$

The pinning matrix $\mathcal{P}$ represents the information flow from the leader, and is $\mathcal{P}=\operatorname{diag}\left\{m_{i 0}, \ldots, m_{N 0}\right\}=I_{N}$, where $I_{N}$ denotes the $(N \times N)$ identity matrix.

Considering the hybrid Predecessor - Leader Following topology, we propose the following control law

$$
\begin{aligned}
u_{i}(t)= & \eta_{i}(t)+k_{3}\left(\eta_{0}-\eta_{i}(t)\right) \\
& +k_{2}\left(q_{0}\left(t-t_{d}\right)-q_{i}\left(t-t_{d}\right)\right) \\
& +k_{1}\left(s_{i-1}\left(t-t_{d}\right)-s_{i}\left(t-t_{d}\right)-d_{r, i}\right)
\end{aligned}
$$

where $k_{1}, k_{2}$, and $k_{3}$ are positive tuning parameters, respectively for position (inter-distance with predecessor), velocity and acceleration (both compared to leader). We assume each vehicle estimates its state from a sensor fusion algorithm, fed from LiDAR, wheel encoders and IMU readings. The control law assumes the state of the leader can be obtained, with delays, from communication link. On the contrary, only the position of the predecessor is obtained through LiDAR.

The time required for data transmission, sensor-to-controller delay, and controller-to-actuator delay are the sources of time delays in this system. These delays can be combined and represented by a single delay. The wireless communication has the largest effect on the whole time delay, and is mainly affected by the number of vehicles inside the platoon. As the number of vehicles varies on a slow timescale, delays can be considered as constant. For analysis purposes, we can assume

Assumption 5. $t_{d}$ is the constant upper bound of the delays.

\section{B. Internal Stability}

Let us define the position error with the leader as $e_{s, i 0}(t)=$ $s_{0}(t)-s_{i}(t)-d_{r, i 0}$, the velocity error with the leader as $e_{q, i 0}(t)=q_{0}(t)-q_{i}(t)$, the acceleration error with the leader as $e_{\eta, i 0}(t)=\eta_{0}(t)-\eta_{i}(t)$, and the position error with the predecessor as $e_{s, i}(t)=s_{i-1}(t)-s_{i}(t)-d_{r, i}$. $e_{s, i}$ can be represented in terms of the error with leader, $e_{s, i 0}$, as $e_{s, i}(t)=e_{s, i 0}(t)-e_{s,(i-1) 0}(t)$. Applying the control law (13) to the system (10), and assuming null jerk $\dot{\eta}_{0} \approx 0$, the error dynamics with regards to the leader yield:

$$
\begin{gathered}
\dot{e}_{s, i 0}(t)=e_{q, i 0}(t), \\
\dot{e}_{q, i 0}(t)=e_{\eta, i 0}(t), \\
\dot{e}_{\eta, i 0}(t)=-\frac{2 k_{1}}{\tau_{i}} e_{s, i 0}\left(t-t_{d}\right)+\frac{k_{1}}{\tau_{i}} e_{s,(i-1) 0}\left(t-t_{d}\right) \\
-\frac{k_{2}}{\tau_{i}} e_{q, i 0}\left(t-t_{d}\right)-\frac{k_{3}}{\tau_{i}} e_{\eta, i 0}(t)
\end{gathered}
$$

Let us define $e_{s, 0}=\left[e_{s, i 0}\right]_{i \in[1, n]}^{T}, e_{q, 0}=\left[e_{q, i 0}\right]_{i \in[1, n]}^{T}$, and $e_{\eta, 0}=\left[e_{\eta, i 0}\right]_{i \in[1, n]}^{T}$ as the position, speed, and acceleration error vectors, respectively, then the error state vector, $X(t)=$ $\left[e_{s, 0}, e_{q, 0}, e_{\eta, 0}\right]^{T}$, is given by

$$
\dot{X}(t)=A_{o} X(t)+A_{d} X\left(t-t_{d}\right),
$$

where $A_{o}$ and $A_{d}$ are represented as

$$
A_{o}=\left[\begin{array}{ccc}
O_{N} & I_{N} & O_{N} \\
O_{N} & O_{N} & I_{N} \\
O_{N} & O_{N} & -k_{3} \mathcal{T}
\end{array}\right], A_{d}=\left[\begin{array}{ccc}
O_{N} & O_{N} & O_{N} \\
O_{N} & O_{N} & O_{N} \\
-k_{1} \mathcal{T} \mathcal{H} & -k_{2} \mathcal{T} & O_{N}
\end{array}\right],
$$

where $O_{N}$ denotes the $(N \times N)$ null matrix, $\mathcal{T}=$ $\operatorname{diag}\left\{\frac{1}{\tau_{1}}, \ldots, \frac{1}{\tau_{N}}\right\} \in \mathbb{R}^{N \times N}$, and $\mathcal{H}=\mathcal{L}+\mathcal{P}$ reflects the topology of the platoon.

From Leibniz-Newton formula, it is known that

$$
X\left(t-t_{d}\right)=X(t)-\int_{-t_{d}}^{0} \dot{X}(t+\epsilon) d \epsilon .
$$

Substituting (17) into (15), given $A_{d}^{2}=0$ from (16) yields:

$$
\dot{X}(t)=A_{a} X(t)-A_{m} \int_{-t_{d}}^{0} X(t+\epsilon) d \epsilon .
$$

where $A_{a}=A_{o}+A_{d}$ and $A_{m}=A_{d} A_{o}$.

Proposition III.1. The matrix $A_{a}$ is Hurwitz stable if and only if $\mathcal{T H}$ has positive eigenvalues with the control gains satisfying the conditions $k_{1}>0, k_{3}>0$, and $k_{2}>\frac{\tau_{i} k_{1} \lambda_{i}}{k_{3}}$.

Proof: The matrix $\mathcal{H}$ is lower-triangular with positive eigenvalues $\lambda_{i}=1$ if $i=1$ and $\lambda_{i}=2$ otherwise. Moreover, $\mathcal{T H}$ also has positive eigenvalues as $\mathcal{T}$ is diagonal with strictly positive terms. According to Schur's formula for commutingblocks matrices [21], the characteristic equation of the matrix $A_{a}$ can be expressed as

$$
\begin{aligned}
\operatorname{det}\left(S I_{3 N}-A_{a}\right) & =\operatorname{det}\left(\left[\begin{array}{ccc}
S I_{N} & -I_{N} & O_{N} \\
O_{N} & S I_{N} & -I_{N} \\
k_{1} \mathcal{T} \mathcal{H} & k_{2} \mathcal{T} & S I_{N}+k_{3} \mathcal{T}
\end{array}\right]\right) \\
& =\prod_{i=1}^{N}\left(S^{3}+\frac{k_{3}}{\tau_{i}} S^{2}+\frac{k_{2}}{\tau_{i}} S+\frac{k_{1}}{\tau_{i}} \lambda_{i}\right) .
\end{aligned}
$$

According to Routh-Hurwitz stability criterion [22], (19) is Hurwitz stable under the conditions $\frac{k_{3}}{\tau_{i}}>0, \frac{k_{1} \lambda_{i}}{\tau_{i}}>0$, and $\frac{k_{2}}{\tau_{i}}>\frac{k_{1} \lambda_{i}}{k_{3}}$, and we previously prove that $\frac{\lambda_{i}}{\tau_{i}}>0$. All eigenvalues of $A_{a}$ thus have negative real parts.

The conditions for the platoon internal stability can be established by the following theorem.

Theorem III.2. Consider a platoon consisting of a leader and $N$ followers, the longitudinal model expressed as (1 - 10), with assumptions 1 - 5, and control (13). Then, the system is asymptotically stable as long as the control gains follow Proposition III.1 and the upper bound of time delay is

$$
t_{d}<\frac{\lambda_{\min }(Q)}{\lambda_{\max }\left(P A_{m} P^{-1} A_{m}^{T} P+b P\right)} .
$$

where $P, Q$ are symmetric positive definite matrices and $b>0$, are detailed in the following lemmas, and $\lambda_{\min }(Q), \lambda_{\max }(Q)$ are the minimum and maximum eigenvalues of $Q$. 
Proof: The proof will mainly depend on the well-known Lyapunov-Razumikhin theorem which is stated as follows.

Lemma III.3. (Lyapunov-Razumikhin Theorem) [23]. Given a system of the form:

$$
\begin{aligned}
\dot{z} & =f\left(z_{t}\right), t>0, \\
z_{0}(\delta) & =\phi(\delta), \forall \delta \in[-\beta, 0],
\end{aligned}
$$

where $z_{t}(\delta)=z(t+\delta), \forall \delta \in[-\beta, 0]$ and $f(0)=0$, the following result holds:

Suppose that the function $f: C\left([-\beta, 0], \mathbb{R}^{n}\right) \rightarrow \mathbb{R}^{n}$ maps bounded sets of $C\left([-\beta, 0], \mathbb{R}^{n}\right)$ into bounded sets of $\mathbb{R}^{n}$. Let $\sigma_{1}, \sigma_{2}$, and $\sigma_{3}$ be continuous, nonnegative, nondecreasing functions with $\sigma_{1}(h)>0, \sigma_{2}(h)>0, \sigma_{3}(h)>0$ for $h>0$ and $\sigma_{1}(0)=\sigma_{2}(0)=0$. If there is a continuous function $V(t, z)$ (Lyapunov-Razumikhin function) such that:

$$
\sigma_{1}(\|z\|) \leq V(t, z) \leq \sigma_{2}(\|z\|), t \in \mathbb{R}, z \in \mathbb{R}^{n},
$$

and there exists a continuous nondecreasing function $\sigma_{4}(h)$ with $\sigma_{4}(h)>h, h>0$ such that

$$
\text { if } \quad \begin{aligned}
\dot{V}(t, z) & \leq-\sigma_{3}(\|z\|), \\
V(t+\delta, x(t+\delta)) & <\sigma_{4}(V(t, x(t))), \delta \in[-\beta, 0],
\end{aligned}
$$

then the solution $z=0$ is uniformly asymptotically stable.

Let $P \in \mathbb{R}^{3 N \times 3 N}$ is a positive definite matrix and consider the following Lyapunov-Razumikhin function

$$
V(X)=X^{T} P X .
$$

Lemma III.4. (Rayleigh-Ritz theorem) [24]. Let a symmetric matrix $Q=Q^{T} \in \mathbb{R}^{n \times n}$ and $z \in \mathbb{R}^{n \times 1}$. Then, we have

$$
\lambda_{\min }(Q)\|z\|^{2} \leq z^{T} Q z \leq \lambda_{\max }(Q)\|z\|^{2},
$$

If one considers $\sigma_{1}(h)=\lambda_{\min }(P) h^{2}$ and $\sigma_{2}(h)=$ $\lambda_{\max }(P) h^{2}$, thus according to Lemma III.4, we have

$$
\sigma_{1}(\|X\|) \leq X^{T} P X \leq \sigma_{2}(\|X\|) .
$$

In addition, note that both $\sigma_{1}(h)$ and $\sigma_{2}(h)$ are continuous, nonnegative, nondecreasing functions with $\sigma_{1}(h)>0$, $\sigma_{2}(h)>0, \sigma_{3}(h)>0$ for $h>0$ and $\sigma_{1}(0)=\sigma_{2}(0)=0$. Therefore, $V(X)$ satisfies condition (22).

Substituting the derivative of $V(X)$ from (18) gives

$$
\dot{V}(X)=X^{T}\left(P A_{a}+A_{a}^{T} P\right) X-2 X^{T} P A_{m} \int_{-t_{d}}^{0} X(t+\epsilon) d \epsilon .
$$

From Proposition III.1, the matrix $A_{a}$ is Hurwitz stable, and hence from Lyapunov theorem, we have

$$
P A_{a}+A_{a}^{T} P=-Q,
$$

where $Q=Q^{T}>0$ and $P=P^{T}>0$.

Lemma III.5. [21]. From the arithmetic-geometric mean inequality, for any positive definite matrix $\mathcal{D}$, it holds

$$
\frac{1}{2}\left(a^{T} \mathcal{D} a+c^{T} \mathcal{D}^{-1} c\right) \geq\left(a^{T} \mathcal{D} a\right)^{\frac{1}{2}}\left(c^{T} \mathcal{D}^{-1} c\right)^{\frac{1}{2}} .
$$

One can also write:

$$
a^{T} c=\left(\mathcal{D}^{\frac{1}{2}} a\right)^{T}\left(\mathcal{D}^{\frac{-1}{2}} c\right) .
$$

Applying the Cauchy-Schwarz inequality to (30) yields:

$$
\begin{aligned}
\left|a^{T} c\right|^{2}=\left|\left(\mathcal{D}^{\frac{1}{2}} a\right)^{T}\left(\mathcal{D}^{\frac{-1}{2}} c\right)\right|^{2} & \leq\left\|\mathcal{D}^{\frac{1}{2}} a\right\|_{2}^{2}\left\|\mathcal{D}^{\frac{-1}{2}} c\right\|_{2}^{2} \\
& \leq\left(a^{T} \mathcal{D} a\right)\left(c^{T} \mathcal{D}^{-1} c\right)^{2}
\end{aligned}
$$

Comparing (31) and (29), we can get:

$$
2 a^{T} c \leq a^{T} \mathcal{D} a+c^{T} \mathcal{D}^{-1} c
$$

Choose $a^{T}=-X^{T} P A_{m}, c=X(t+\epsilon), \mathcal{D}=P^{-1}$, and integrating both side of the inequality, we have along (28)

$$
\begin{aligned}
\dot{V}(X) \leq & -X^{T} Q X+t_{d} X^{T} P A_{m} P^{-1} A_{m}^{T} P X \\
& +\int_{-t_{d}}^{0} X^{T}(t+\epsilon) P X(t+\epsilon) d \epsilon .
\end{aligned}
$$

According to Lemma III.3, let us choose the following continuous nondecreasing function $\sigma_{4}(h)=b h$ (for some constant $b>1$ ), such that the condition in (III.3) is satisfied and hence we have

$$
\begin{aligned}
V(X(t+\epsilon)) & =X^{T}(t+\epsilon) P X(t+\epsilon) \\
& \leq \sigma_{4}(V(X)) \\
& =b V(X) \\
& =b X^{T}(t) P X(t) .
\end{aligned}
$$

Substituting from (34) into (33) and solving the integral, we have

$$
\begin{aligned}
\dot{V}(X) & \leq-X^{T} Q X+t_{d} X^{T} P A_{m} P^{-1} A_{m}^{T} P X+t_{d} b X^{T} P X \\
& \leq-\lambda_{\min }(Q)\|X\|^{2}+t_{d} \lambda_{\max }\left(P A_{m} P^{-1} A_{m}^{T} P+b P\right)\|X\|^{2} \\
& \leq-\sigma_{3}(\|X\|)
\end{aligned}
$$

Let us define

$$
\sigma_{3}(\|X\|)=\left(\lambda_{\min }(Q)-t_{d} \lambda_{\max }\left(P A_{m} P^{-1} A_{m}^{T} P+b P\right)\right)\|X\|^{2}
$$

which is a continuous nonnegative nondecreasing function provided that it is constrained by inequality (20). Therefore, condition (III.3) of $\dot{V}(X)$ is also satisfied. Consequently, the asymptotic stability of the tracking error $X(t)$ is achieved.

\section{String Stability}

The platoon controller must guarantee string stability; otherwise, the errors may amplify when propagating downstream the vehicle string, which may result in rear-end collision.

Definition III.6. $L_{2}$ String Stability means that the energy (with $L_{2}$ norm) of the position error $e_{s, i}$ of vehicle $i$, is smaller than the energy of the position error of its predecessor [25]. The string stability is thus guaranteed if $\|H(s)\|_{\infty}<1$, since

$$
\|H(S)\|_{\infty}=\sup _{e_{s, i-1} \in L_{2}} \frac{\left\|e_{s, i}\right\|_{2}}{\left\|e_{s, i-1}\right\|_{2}} .
$$

For simplicity, let us define the following errors $e_{i}(t)=$ $s_{i-1}(t)-s_{i}(t)-d_{r, i}, \dot{e}_{i}(t)=q_{i-1}(t)-q_{i}(t), e_{i 0}(t)=s_{0}(t)-$ $s_{i}(t)-d_{r, i 0}, \dot{e}_{i 0}(t)=q_{0}(t)-q_{i}(t)$, and $\ddot{e}_{i}(t)=\eta_{i-1}(t)-\eta_{i}(t)$. 
It is known that the vehicles involved in the car sharing system are often with the same type of small passenger vehicles, or have very close dynamics, thus we can assume

Assumption 6. All the vehicles are assumed to have equal dynamics, i.e., $\tau_{i}=\tau \forall i=1,2, \ldots, N$.

From (10 and 13), considering the relation between the errors with leader and those with the predecessor, and after some algebraic derivations, one can find the following expression,

$$
\begin{gathered}
\dddot{e}_{i}(t)=\dot{\eta}_{i-1}(t)-\dot{\eta}_{i}(t), \\
\tau \dddot{e}_{i}(t)=-k_{3} \ddot{e}_{i}(t)-2 k_{1} e_{i}\left(t-t_{d}\right)+k_{1} e_{i-1}\left(t-t_{d}\right) \\
-k_{2} \dot{e}_{i}\left(t-t_{d}\right) .
\end{gathered}
$$

Writing (36) in the S-domain gives

$$
G(S)=\frac{E_{i}(S)}{E_{i-1}(S)}=\frac{k_{1} e^{-t_{d} S}}{\tau S^{3}+k_{3} S^{2}+k_{2} S e^{-t_{d} S}+2 k_{1} e^{-t_{d} S}} .
$$

Theorem III.7. Consider the platoon system presented in Theorem III.2, with assumptions 1 - 6, and the control algorithm proposed in (13) is applied to it. Then, the platoon is string stable if the following conditions are satisfied

$$
\begin{aligned}
& k_{2}^{2}-4 k_{1} k_{3}>0, \\
& t_{d}<\frac{k_{3}^{2}-2 k_{2} \tau}{2 k_{2} k_{3}-4 k_{1} \tau}, \\
& k_{3}^{2}-2 k_{2} \tau>0 \\
& k_{2} k_{3}-2 k_{1} \tau>0 .
\end{aligned}
$$

Proof: In the frequency-domain, the transfer function, $G(S)$, in (37), is given by

$$
G(j w)=\frac{k_{1} e^{-t_{d}(j w)}}{\tau(j w)^{3}+k_{3}(j w)^{2}+k_{2}(j w) e^{-t_{d}(j w)}+2 k_{1} e^{-t_{d}(j w)}} .
$$

If one can prove that $|G(j w)|=\sqrt{\frac{g_{n}(w)}{g_{d}(w)}}<1$, or $g_{d}(w)-$ $g_{n}(w)>0, \forall w>0$, then the condition $\|G(S)\|_{\infty}<1$ holds.

After some algebraic manipulations, one can get

$$
\begin{aligned}
g_{d}-g_{n}= & \tau^{2} w^{6}+\left(k_{3}^{2}-2 k_{2} \tau \cos \left(t_{d} w\right)\right) w^{4} \\
& +\left(\left(4 k_{1} \tau-2 k_{2} k_{3}\right) \sin \left(t_{d} w\right)\right) w^{3} \\
& +\left(k_{2}^{2}-4 k_{1} k_{3} \cos \left(t_{d} w\right)\right) w^{2}+3 k_{1}^{2} .
\end{aligned}
$$

Exploiting the fact $-\cos (z) \geq-1$, it follows

$$
\begin{aligned}
k_{3}^{2}-2 k_{2} \tau \cos \left(t_{d} w\right) & \geq k_{3}^{2}-2 k_{2} \tau, \\
k_{2}^{2}-4 k_{1} k_{3} \cos \left(t_{d} w\right) & \geq k_{2}^{2}-4 k_{1} k_{3} .
\end{aligned}
$$

Moreover, given that $-\sin (z) \geq-z, \forall z \geq 0$, the bound of $t_{d}$ must be positive, and given $k_{2}>\frac{2 k_{1} \tau}{k_{3}}$ from the internal stability conditions (see Proposition III.1 and Theorem III.2), it follows that $\left(4 k_{1} \tau-2 k_{2} k_{3}\right)<0$, and hence one can deduce

$$
\left(4 k_{1} \tau-2 k_{2} k_{3}\right) \sin \left(t_{d} w\right) \geq\left(4 k_{1} \tau-2 k_{2} k_{3}\right) t_{d} w .
$$

Substituting (41 and 42) into (40), after some algebraic manipulations, we have

$$
\begin{aligned}
g_{d}-g_{n} \geq & \tau^{2} w^{6}+\left(k_{3}^{2}-2 k_{2} \tau+4 k_{1} \tau t_{d}-2 k_{2} k_{3} t_{d}\right) w^{4} \\
& +\left(k_{2}^{2}-4 k_{1} k_{3}\right) w^{2}+3 k_{1}^{2} .
\end{aligned}
$$

Investigating (43), the constraint $g_{d}-g_{n}>0$, and consequently $|G(j w)|<1, \forall w>0$, is satisfied if the conditions (38a and 38b) are hold.

Given $2 k_{2} k_{3}-4 k_{1} \tau>0$ from Proposition III.1 and Theorem III.2, one can set another condition (38c).

Corollary III.8. The presented platoon control system is asymptotically stable and string stable provided that the control gains are positive and the following conditions hold

$$
\begin{aligned}
& k_{2}<k_{3}^{2} /(2 \tau) \\
& k_{1}<\min \left\{\frac{k_{2}^{2}}{4 k_{3}}, \frac{k_{2} k_{3}}{\tau \lambda_{i}}\right\}, \lambda_{i}= \begin{cases}1 & \text { if } i=1 \\
2 & \text { otherwise }\end{cases} \\
& t_{d}<\min \left\{\frac{k_{3}^{2}-2 k_{2} \tau}{2 k_{2} k_{3}-4 k_{1} \tau}, \frac{\lambda_{\min }(Q)}{\lambda_{\max }\left(P A_{m} P^{-1} A_{m}^{T} P+b P\right)}\right\} .
\end{aligned}
$$

Proof: After some algebraic manipulations, this corollary can be proved by checking the conditions given in Proposition III.1, Theorems III.2 and III.7.

\section{RESUlts AND Discussions}

The proposed control strategy implemented in a vehicular mobility simulator, based on the Robot Operating System (ROS) with the following features:

- full vehicle dynamics;

- real maps of an urban environment (our campus);

- both low and high level measurement units with noises;

- low level communication module between different parts of the vehicle and the on-board computer;

- vehicle communication modules;

- Human-Machine Interface to send high-level commands to the vehicle in either simulation or real-time.

The platoon is composed of $N$ followers (Renault ZOE) plus a manually driven leader (Renault FLUENCE). The parameters of the vehicles are given in Table I. The parameter $\tau$ is identified from experimental tests.

The platoon is tested inside the Centrale Nantes (ECN) campus, the map of which is initially available in all cars.

The vehicles are initially parked. While the leader moves, its path is constructed online and broadcasted to the followers.

The lateral controller proposed in [3] is in charge of producing the steering angle of the vehicle (by minimizing both the lateral and angular deviations, $r_{i}$ and $\psi_{i}$, respectively).

The longitudinal controller is implemented at a rate of $100 \mathrm{~Hz}$. Control parameters (Table II) guarantee the stability given in Corollary III.8. The theoretical upper bound of time delay, satisfying the condition given in (44c), is $12.443 \mathrm{~ms}$. For safety and comfortability, the vehicle velocity and acceleration are constrained as given in Table II. 
Table I

VEHICLES PARAMETERS

\begin{tabular}{|c|c|c|}
\hline Parameter/Vehicle & FLUENCE & ZOE \\
\hline Mass & $1605 \mathrm{~kg}$ & $1428 \mathrm{~kg}$ \\
\hline Width & $1.545 \mathrm{~m}$ & $1.945 \mathrm{~m}$ \\
\hline Height & $1.462 \mathrm{~m}$ & $1.562 \mathrm{~m}$ \\
\hline Length & $4.748 \mathrm{~m}$ & $4.084 \mathrm{~m}$ \\
\hline Rear-axis to Rear-end & $1.140 \mathrm{~m}$ & $0.657 \mathrm{~m}$ \\
\hline distance between axes & $2.701 \mathrm{~m}$ & $2.588 \mathrm{~m}$ \\
\hline distance between wheels & $1.545 \mathrm{~m}$ & $1.511 \mathrm{~m}$ \\
\hline Wheel radius & $0.29 \mathrm{~m}$ & $0.29 \mathrm{~m}$ \\
\hline Inertia momentum & $45.0 \mathrm{kgm}^{2}$ & $28.0 \mathrm{kgm}^{2}$ \\
\hline$\tau$ & - & 0.2 \\
\hline
\end{tabular}

Table II

CONTROLLER PARAMETERS FOR BOTH SIMULATION AND EXPERIMENTS

\begin{tabular}{|c|c|c|c|}
\hline Parameter & Value & Parameter & Value \\
\hline$u_{i}$ & $\in[-6,1] \mathrm{m} / \mathrm{s}^{2}$ & $v_{i}$ & $\in[0,8] \mathrm{m} / \mathrm{s}$ \\
\hline$d_{r, i}$ & $10 \mathrm{~m}$ & $k_{3}$ & 0.400 \\
\hline$k_{2}$ & 0.380 & $k_{1}$ & 0.018 \\
\hline$b$ & 1.1 & $Q$ & $I_{3 N}$ \\
\hline
\end{tabular}

\section{A. Simulation Results}

Three followers are considered in simulation. The reference and actual trajectories are shown in Fig. 6. Figures 7e and $7 \mathrm{f}$ present lateral and angular deviations and illustrate the capabilities of the lateral controller.

Figures $7 \mathrm{a}-7 \mathrm{~d}$ show the longitudinal behavior. The leader travels with variable velocity, see Fig. $7 \mathrm{~b}$. The vehicles start from distances different from the desired inter-vehicle distance and reach the consensus after about $10 \mathrm{~s}$.

Table III

RMSE OF TRACKING ERRORS IN SIMULATION.

\begin{tabular}{|c|c|c|c|c|}
\hline Follower & $e_{s, i}[m]$ & $e_{q, i}[\mathrm{~m} / \mathrm{s}]$ & $r_{i}[\mathrm{~m}]$ & $\psi_{i}[\mathrm{rad}]$ \\
\hline 1 & 0.2103 & 0.0763 & 0.0455 & 0.0092 \\
\hline 2 & 0.0872 & 0.0297 & 0.0439 & 0.0091 \\
\hline 3 & 0.0482 & 0.0219 & 0.0441 & 0.0087 \\
\hline
\end{tabular}

Moreover, the followers achieve consensus in case of accelerations perturbations, see Fig. 7a. Table III presents the Root Mean Squared Error (RMSE) of the tracking errors which are in the acceptable ranges. The position tracking errors are attenuated as the RMSE of $e_{s, 3}<e_{s, 2}<e_{s, 1}$, which enlightens the string stability of the platoon.

\section{B. Experimental Results}

For real experiments, we use two Renault ZOE followers, see Fig. 8. Each one is equipped with a radio module (ARM-N8-SIGFOX with $868 \mathrm{Mhz} 1 / 2$ wave antenna), an IMU (XSENS MTI-100), a GPS-RTK (PROFLEX-800), a LiDAR

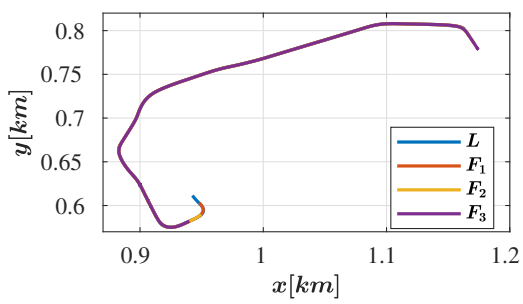

Figure 6. Path tracking; $L$ : leader actual path, and $F_{i}$ : follower $i$ actual path. The followers track the path perfectly.

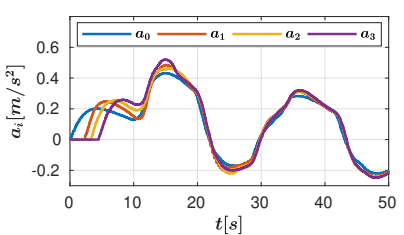

(a) Acceleration perturbation

(c) Position tracking

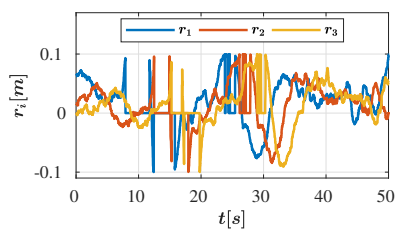

(e) Path tracking: lateral error

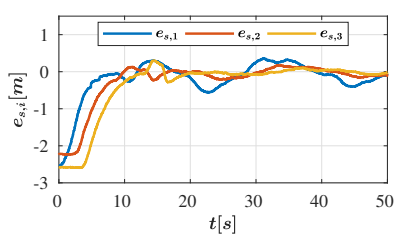

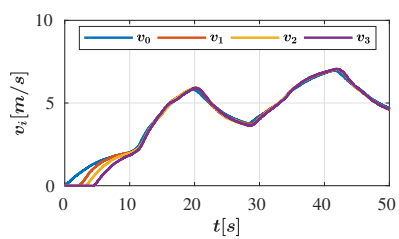

(b) Variable velocity

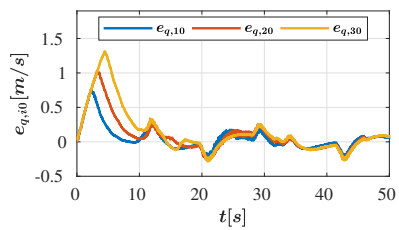

(d) Velocity tracking

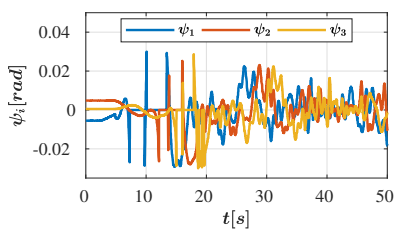

(f) Path tracking: orientation error
Figure 7. Platoon simulation results.

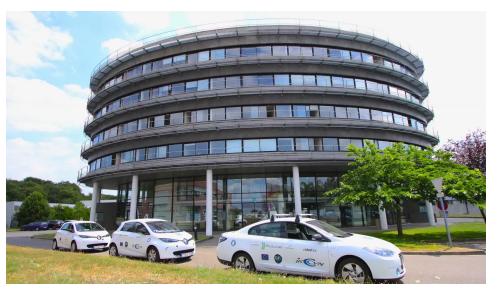

Figure 8. Platoon prototype in ECN campus.

(Velodyne VLP-16), and an onboard Computer (Doliath 1000 - Intel Core i5-3610ME 2.10GHz). The software architecture is developed in C++ with ROS Kinetic on Ubuntu 16.04.

Figures $9 \mathrm{e}$ and $9 \mathrm{f}$ illustrate the tracking capabilities of the lateral controller. Figures $9 \mathrm{a}-9 \mathrm{~d}$ illustrate the longitudinal behavior with a manually-driven leader and accelerations perturbations (see Fig. 9a). Table IV presents the Root Mean Squared Error (RMSE) of the tracking errors. They are larger than in the simulation study but still in the acceptable ranges. Finally, string stability is validated as the RMSE of $e_{s, 2}<e_{s, 1}$. The full studies are depicted in this video. 


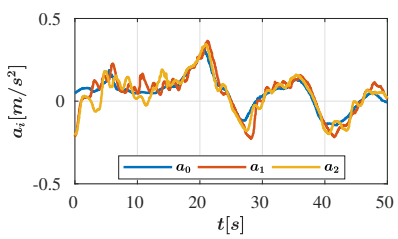

(a) Acceleration perturbation

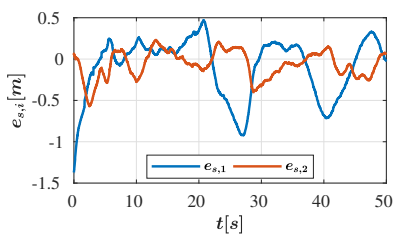

(c) Position tracking

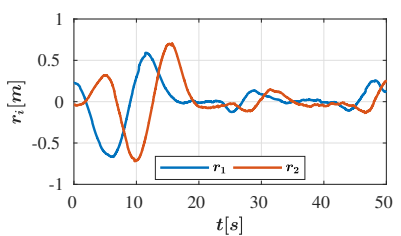

(e) Path tracking: lateral error

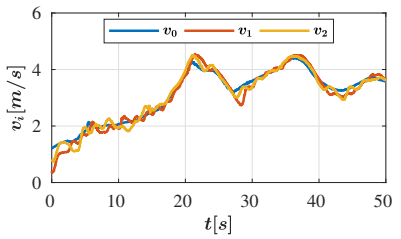

(b) Variable velocity

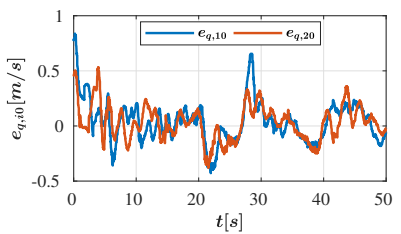

(d) Velocity tracking

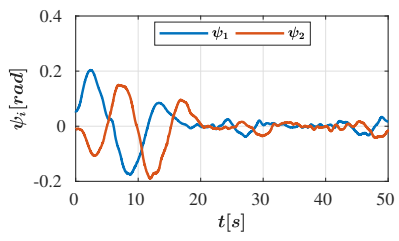

(f) Path tracking: orientation error
Figure 9. Platoon experimental results.

Table IV

RMSE OF TRACKING ERRORS IN REAL-TIME

\begin{tabular}{|c|c|c|c|c|}
\hline RMSE & $e_{s, i}[\mathrm{~m}]$ & $e_{q, i}[\mathrm{~m} / \mathrm{s}]$ & $r_{i}[\mathrm{~m}]$ & $\psi_{i}[\mathrm{rad}]$ \\
\hline 1 & 0.3416 & 0.3189 & 0.0794 & 0.0194 \\
\hline 2 & 0.1306 & 0.1576 & 0.3801 & 0.0909 \\
\hline
\end{tabular}

\section{CONCLUSIONS}

This paper presents a successful application of a distributed third-order longitudinal controller for a platoon in an urban environment. We assume a urban environment induces high curvature path, hence only the predecessor's position is measured by an onboard sensor (LiDAR). On the opposite, the full state of the leader is obtained through communication link. Conditions for both platoon internal and string stability are given, under the assumption that all vehicles have the same dynamics. Stability amounts to impose a higher bound on the overall time delay of the system. The efficiency of the proposed approach are demonstrated both in simulation and experimental studies, with a leader and two followers.

\section{ACKNOWLEDGMENT}

This work was supported by the Valet and ROBOTEX projects, reference ANR-15-CE22-0013-02 and ANR-10EQPX-44-01. Moreover, we thank Prof. G. Garcia and Dr. A. Hamon, for their involvement during the experiments.

\section{REFERENCES}

[1] D. Swaroop, J. Hedrick, C. Chien, and P. Ioannou, "A comparision of spacing and headway control laws for automatically controlled vehicles1,"Vehicle Syst. Dyn., vol. 23, no. 1, pp. 597-625, 1994.
[2] J. Bom, B. Thuilot, F. Marmoiton, and P. Martinet, "A global control strategy for urban vehicles platooning relying on nonlinear decoupling laws," in IEEE/RSJ Int. Conf. on Intelligent Robots and Systems, 2005, pp. 2875-2880.

[3] B. Thuilot, J. Bom, F. Marmoiton, and P. Martinet, "Accurate automatic guidance of an urban electric vehicle relying on a kinematic gps sensor," IFAC Proc. Volumes, vol. 37, no. 8, pp. 155-160, 2004.

[4] S. E. Li, Y. Zheng, K. Li, and J. Wang, "An overview of vehicular platoon control under the four-component framework," in IEEE Intelligent Vehicles Symp., 2015, pp. 286-291.

[5] J.-W. Kwon and D. Chwa, "Adaptive bidirectional platoon control using a coupled sliding mode control method," IEEE Trans. Intell. Transp. Syst., vol. 15, no. 5, pp. 2040-2048, 2014.

[6] W. Yue, G. Guo, L. Wang, and W. Wang, "Nonlinear platoon control of arduino cars with range-limited sensors," Int. Journal of Control, vol. 88, no. 5, pp. 1037-1050, 2015.

[7] F. Lin, M. Fardad, and M. R. Jovanovic, "Optimal control of vehicular formations with nearest neighbor interactions," IEEE Trans. on Automatic Control, vol. 57, no. 9, pp. 2203-2218, 2012.

[8] R. Lemos, O. Garcia, and J. V. Ferreira, "Local and global path generation for autonomous vehicles using splines," Ingeniería, vol. 21, no. 2, pp. 188-200, 2016.

[9] M. di Bernardo, P. Falcone, A. Salvi, and S. Santini, "Design, analysis, and experimental validation of a distributed protocol for platooning in the presence of time-varying heterogeneous delays," IEEE Trans. on Control Systems Technology, vol. 24, no. 2, pp. 413-427, 2016.

[10] J. C. Zegers, E. Semsar-Kazerooni, J. Ploeg, N. van de Wouw, and H. Nijmeijer, "Consensus control for vehicular platooning with velocity constraints," IEEE Trans. on Control Systems Technology, 2017.

[11] P. Martinet, B. Thuilot, and J. Bom, "From autonomous navigation to platooning in urban context," in Proc. of The Iarp-workshop on Adaptive and Intelligent Robots: Present and Future, vol. 1, no. 1, 2005, pp. 1-9.

[12] S. E. Li, H. Peng, K. Li, and J. Wang, "Minimum fuel control strategy in automated car-following scenarios," IEEE Trans. on Vehicular Technology, vol. 61, no. 3, pp. 998-1007, 2012.

[13] J. VanderWerf, S. Shladover, N. Kourjanskaia, M. Miller, and H. Krishnan, "Modeling effects of driver control assistance systems on traffic," Transportation Research Record: Journal of The Transportation Research Board, no. 1748, pp. 167-174, 2001.

[14] H. Chehardoli and M. R. Homaeinezhad, "Third-order leader-following consensus protocol of traffic flow formed by cooperative vehicular platoons by considering time delay: constant spacing strategy," Proc. Inst. Mech. J. Syst. Control Eng., vol. 232, no. 3, pp. 285-298, 2018.

[15] M. Fardad, F. Lin, and M. R. Jovanović, "Sparsity-promoting optimal control for a class of distributed systems," in American Control Conf., 2011, pp. 2050-2055.

[16] Y. Wu, S. E. Li, Y. Zheng, and J. K. Hedrick, "Distributed sliding mode control for multi-vehicle systems with positive definite topologies," in IEEE Conf. on Decision and Control, 2016, pp. 5213-5219.

[17] W. B. Dunbar and D. S. Caveney, "Distributed receding horizon control of vehicle platoons: Stability and string stability," IEEE Trans. on Automatic Control, vol. 57, no. 3, pp. 620-633, 2012.

[18] S. Santini, A. Salvi, A. S. Valente, A. Pescapé, M. Segata, and R. L. Cigno, "A consensus-based approach for platooning with intervehicular communications and its validation in realistic scenarios," IEEE Trans. on Vehicular Technology, vol. 66, no. 3, pp. 1985-1999, 2017.

[19] M. di Bernardo, A. Salvi, and S. Santini, "Distributed consensus strategy for platooning of vehicles in the presence of time-varying heterogeneous communication delays," IEEE Trans. Intell. Transp. Syst., vol. 16, no. 1, pp. 102-112, 2015 .

[20] A. Khalifa, O. Kermorgant, S. Dominguez, and P. Martinet, "Vehicles platooning in urban environment: Consensus-based longitudinal control with limited communications capabilities," in Int. Conf. on Control, Automation, Robotics and Vision, 2018.

[21] R. A. Horn and C. R. Johnson, Matrix analysis. Cambridge university press, 2012

[22] R. C. Dorf and R. H. Bishop, Modern control systems. Pearson, 2011.

[23] J. K. Hale and S. M. V. Lunel, Introduction to functional differential equations. Springer Science \& Business Media, 2013, vol. 99.

[24] J. W. Demmel, Applied numerical linear algebra. Siam, 1997, vol. 56.

[25] J. Eyre, D. Yanakiev, and I. Kanellakopoulos, "A simplified framework for string stability analysis of automated vehicles ," Vehicle System Dynamics, vol. 30, no. 5, pp. 375-405, 1998. 